\title{
The Use of Generative Modelling to Automate the Design of Aerial Structural Assemblies
}

\author{
Wojciech SKARKA ${ }^{1}$ and Mikir ALEGAZE \\ Silesian University of Technology, Faculty of Mechanical Engineering, Poland
}

\begin{abstract}
This paper presents a method of automating design that has the potential to significantly improve the design process. The intent is to develop a design automation system that is an integration of several methods in a holistic approach for use. The automation Generative Design approach allows the reuse of wing design data and parameters. For this purpose, Generative Design methodologies are discovered to enhance the system building. This example of an analyzed wing structure to develop a compatible method to automate the design is described. The model parameterization technique is used to facilitate the generative model creation based on parameters, variables, and constraints. The use of integration in SolidWorks software function is proposed as part of an approach to support designers during both the generative model-building phase of the design process and simulation verification phase. Finally, an example and validation process is presented. The aim is to quickly and cost-effectively validate analytical results from the developed process. The proposed process adopts flexibility to acquire the intended model on different sizes and purposes, likewise, it is shown how the implemented approach makes it possible to automate the generation of generative models for FEM analysis.
\end{abstract}

Keywords. Generative Model, wing design, design automation, aircraft design, simulation, transdisciplinary engineering

\section{Introduction}

Due to the existing competition in Aerospace companies, engineers in design and development departments attempt to minimize cost and time consumption for wing designs. Consequently, a need for integrated tools and methods throughout the development process has been raised. To help the design process in reducing design challenges, time, and cost. Integration of various disciplines in the wing preliminary design as one entity enables the design process to be kept intact at every step.

Automating design is a promising method to solve these problems and significantly improve the design process. During the design process, numerous variants, alternatives, and possible solutions are explored and compared iteratively, until the product is found that best suits the requirements. Moreover, changes to the design can come after the product has hit the market, as further developments, derivatives, and updates are requested. Complying with and implementing all the changes is a very time-consuming and costly activity [1]. Enabling automation of as many as possible of the repetitive

\footnotetext{
${ }^{1}$ Corresponding Author, Mail: wojciech.skarka@polsl.pl.
} 
processes during product development is a key to increasing productivity, reducing timeto-market, and cutting costs. Additionally, freeing designers from tedious work allows them to concentrate on activities that require creativity, which is hardly possible to integrate into a computer system. The intent is to develop a design automation system that is an integration of several methods in a holistic approach for use. The automation Generative Design approach allows the reuse of wing design data and parameters. For this purpose, Generative Design methodologies are discovered to enhance the system building.

Aircraft design is a multi-disciplinary and time-consuming process that involves a large number of disciplines and expertise in flight controls, structures aerodynamics, propulsion, and systems amongst others. During the initial conceptual stage of an aircraft design process, multiple aircraft configurations are studied and analyzed. Feasibility studies for various concepts and designs are carried out and the goal is to come up with a design concept that can best achieve the design objectives. One of the vital studies in any aircraft design process is the preliminary design study of an aircraft wing. The aircraft wing is a critical design component of an aircraft from a structural and aerodynamics point of view.

\section{State of the art}

The generative design was started by Frazer [2] in the early 1970s. It developed to some extent mostly focused on design theory, although there was no implementation method [3], due to the rising interest of engineers, this gap was filled by CAD companies [3, 4, 5,6 ,], offering various generative design solutions. The current aim of generative design is best summed up by Shea; "generative design systems are aimed at creating new design processes that produce spatially novel yet efficient and buildable designs through the exploitation of current computing and manufacturing capabilities" [7].

A particular form of generative design - the Generative Design Method (GDM) [8] derived from Knowledge-Based Engineering [9], is defined here as - a designer driven, parametrically constrained design exploration process, operating on top of history-based parametric CAD systems that are unstructured in terms of design development and supportive of emergence [10]. A wide range of methods and modeling techniques were developed till now: Constraint-Based Evolutionary Decision Support System [11], GENE_ARCH [12], Intelligent Genetic Design Tool [13], Genetic Algorithm Designer (GADES) [14], Shape Grammar [15]

The Generative Modeling (GM) method belongs to the group of generative methods, ie the Generative Design Method (GDM). There is also one of the methods of Knowledge-Based Engineering (KBE) and due to the intensive use of geometric modeling, it is one of the methods of Geometry-Based Design Automation (GBDA) [16] Such systems are particularly suitable for design families of highly similar structures [8, 17, 18] or modular systems [19] but it can be used in other more sophisticated applications from aerospace [16] automotive [20,21] or a manufacturing industry [22]. Advanced applications of Generative Modeling in optimization, in particular in Multidisciplinary Design Optimization (MDO), are found more and more often [16, 23].

The Generative Design Method (GDM) is an inclusive CAD-based generative design study method designed suitable at all stages of the design development process starting from conceptual to detailed design. The GDM comprises six key components: $[8,17]$ 
1. Genotype - is composed of a generic parametric CAD model, a list of design parameters and their initial value, and an initial exploration envelope.

2. Phenotype - generated CAD files (that may include build history, built-in relationships, and built-in equations).

3. Exploration envelope (Constraints) - a list of minimum and maximum values of the driving parameters specifying the limits of the design space to be explored.

4. Design Table - a data table that stores the driving design parameters, their initial values, and the limits, and other data that may be required, and the generated design values preferable in an accessible spreadsheet format.

5. Design Generation Macro - a macro or a spreadsheet function that operates on the design table. It generates random variations of the driving parameters within limits set by the initial design envelope.

6. CAD system - is a parametric CAD engine with a transparent and editable build history, preferably with a 3D geometric kernel with capabilities to manage geometric relationships, engineering equations and connect to external design tables.

7. Performance filters - A pass/fail software filter, that can evaluate the performance of generated designs based on data from the design table, CAD system or associated analytical packages.

\section{Generative modeling methodology}

\subsection{Structure}

The knowledge-based method combined structural design and Generative Model designing system are used thoroughly including several sub-systems to optimize the whole process.

The generative models building approach started by establishing an inter-related discipline and sub-systems vary from data acquisition, design calculation, design automation, CAD, and FEA.

The development started in specifying the wing conceptual design criteria and parameters. Basic wing structural analyses are performed on strength and aerodynamic perspective. Few parameters of wing component geometries are linked to sizing analysis results through the design table. Any updates in the level of the parameter will affect the CAD model, likewise, the initial design criteria are linked with the generative model building approach. The approach uses both parameters and analysis to implement in CAD model building. Moreover, Wing structure assembly is made in relation to wing structural configurations analysis and result through the developed generative model building approach. The system will allow a generation of different types of wing configurations as required directly from given parameters.

Then, the testing of the generic model was done to ensure that the generic aircraft wing model can cater to different types of aircraft wing configurations and planform shapes. The next step included the use of generic model components assembly and making structural mesh generation and making FEA of the generic aircraft wing model. Eventually, FEA can be performed at any part or assembly configuration that was made by a generative model. The application of the model follows a new design paradigm with a generative model (Figure 1). The problem with the new approach is the integration of the results of the following analyzes in the design process, and thus in the set of rules controlling the construction of Generative Models. Distribution of aerodynamic loads on 
the parameter resulting from flow analysis, an envelope of loads resulting from operational states of the airplane. Strength analyzes resulting from the general issues of the strength of materials as well as specific ones related to, for example, the design of composite material, the installation layout resulting from the structure and construction of various specialized installations on the plane, specialized rules, and good aviation practices (aeroelasticity, handling), the technology of manufacturing basic aircraft structures. These are various fields and the ongoing integration of the analysis results guarantees a transdisciplinary approach. The tasks to be performed and the relevant parameters that are the input to the next steps are described below.

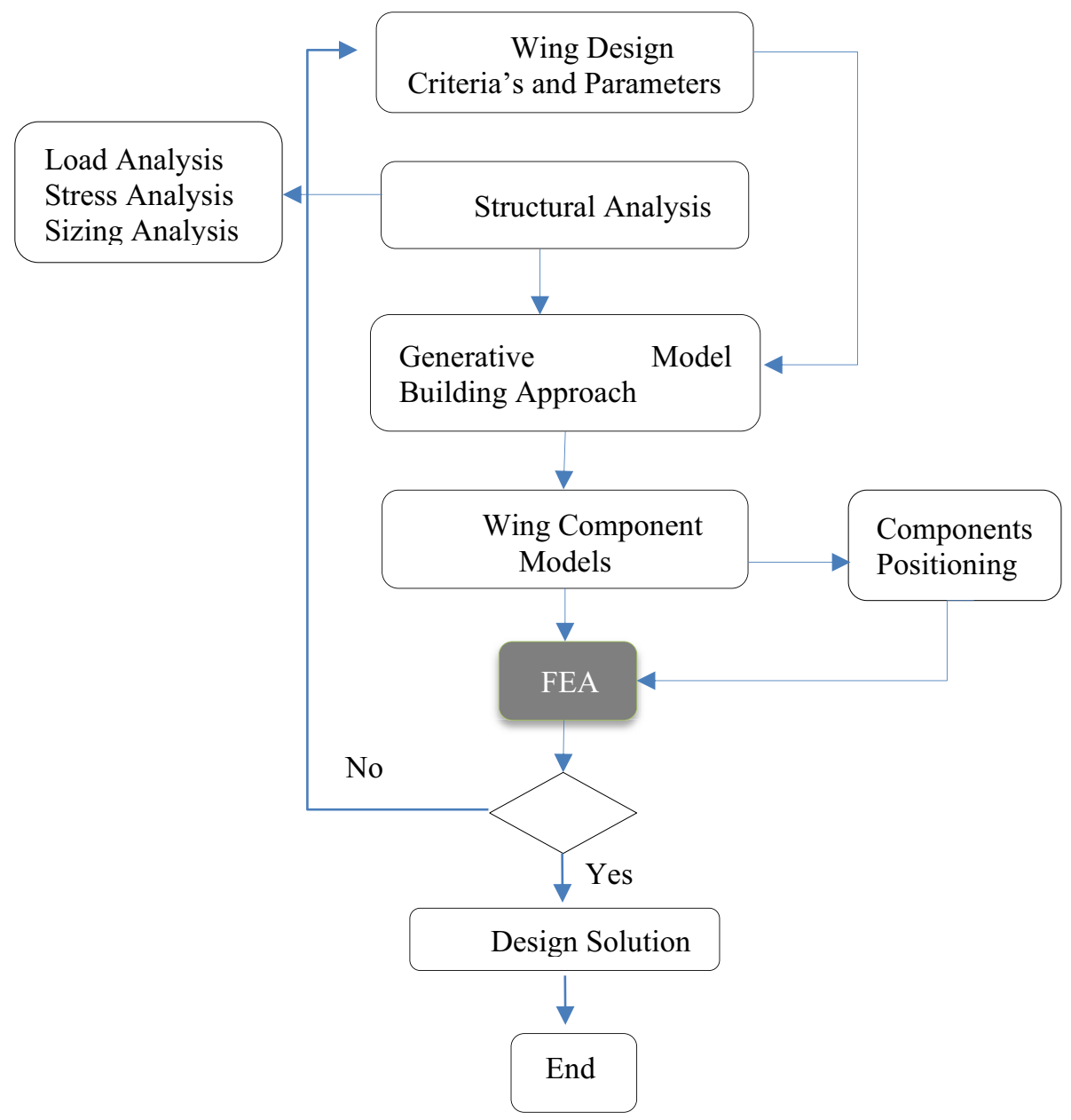

Figure 1. The paradigm of Automation of Wing Design using Generative Modeling Method.

\subsubsection{Wing Design Parameters}

This wing design analysis began with choosing the aircraft application. The geometrical and aerodynamical parameters of the chosen aircraft are specified for structural analysis. 
According to these assumptions, the basic features of the wing are selected according to which the conceptual structure of the wing can be proposed (Figure 2, Figure 3).

Aspect Ratio- is defined as the wingspan squared divided by the wing area.

Taper ratio- defined as the ratio between the tip chord of the wing and the root chord of the wing.

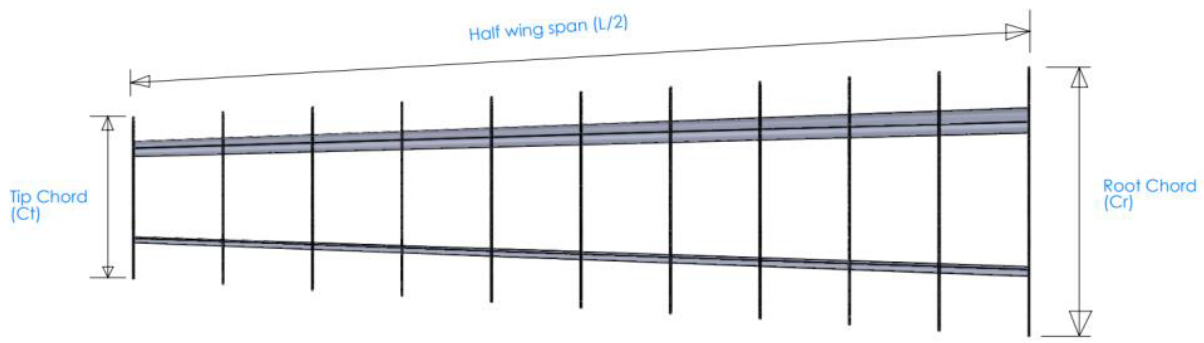

Figure 2. Swept wing geometrical parameters.

Mean Aerodynamic Chord- is the average chord length of a tapered or swept wing. Airfoil Shape- is defined as coordinates that form the shape of an airfoil and wing ribs.

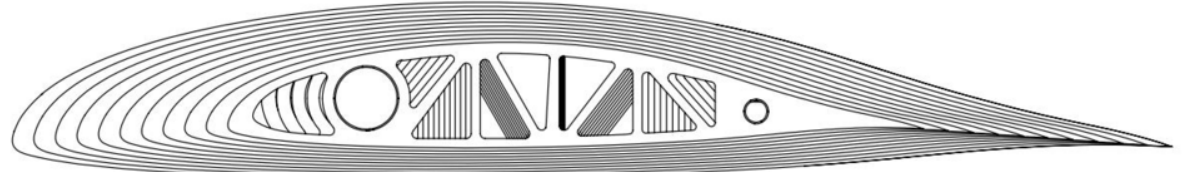

Figure 3. Wing rib shape.

\subsubsection{Wing Load Analysis}

Wing load analyses are a very crucial beginning. Wing geometrical parameters are used to analyze three major load analyses of aircraft wings. Obtaining different results are possible by changing geometrical parameters, which later influences sizing analysis of spar or rib.

\subsubsection{Sizing and Stress Analysis}

Sizing analysis is dedicated to determining wing components thickness based on calculation results, which are simultaneously linked to CAD through the generative modeling system. Thus, various sizes are obtained at different initial design criteria and loads.

\subsubsection{Generative Modeling Approach}

Later wing structural analysis, various wing component sizing is calculated hence become one of the major roles in determining generic models. The concept of the wing components generic model was then developed in the generative method approach. 
Spar positioning and ribs spacing concepts are developed similarly as wing components to generate an assembly generic model.

The theoretical outlines for the proposed generative model design method alongside methods of implementation are presented in Figure 4.

The methods of building a generative model process involve relations of various software and techniques. Wing geometrical parameters are considered as the initial step to build fixed geometries, while each parameter are interrelated with SolidWorks function, such as formulas and constraints. The thickness of components and wing structural assemblies are regulated by a design table where these parameters are linked to excel calculation. Thus, the whole approach allows a generation of different wing model configurations that can also be automatically updated in FEA.

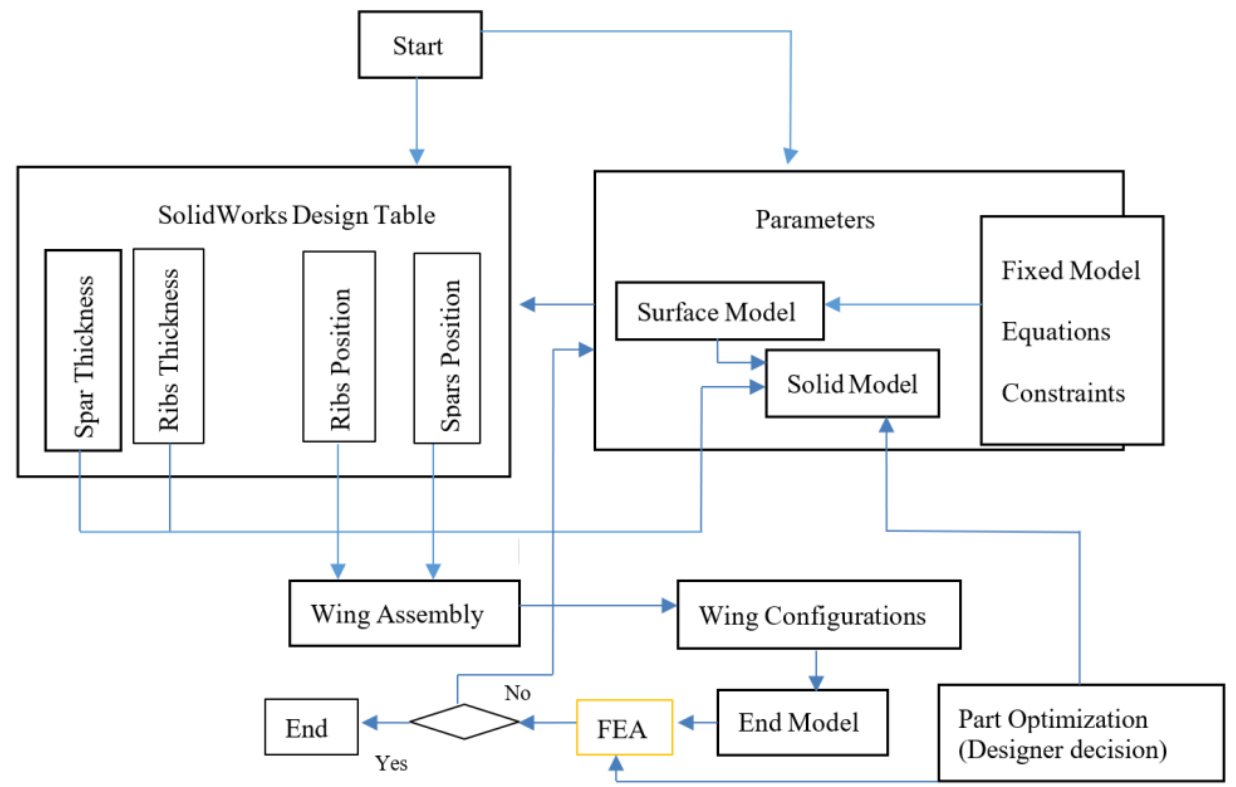

Figure 4. The paradigm of Generative Model Building.

\subsubsection{Concept of Wing components Generative Model Building}

The spar building method has contained few constraints, formulas, and equation relations which are mostly geometrical and thickness as shown in Table 1. Furthermore, geometrical parameters are directly linked to the calculation analysis, hence iteration is achievable in the calculation spreadsheet directly affects the thickness.

Ribs parameters include airfoil coordinates, shape and chord length, Web length, flange width, diameter for circular section thickness, material, and color. Thickness is directly driven from a spreadsheet calculation result, as a result, different CAD model outputs are obtained in an iteration. 
Table 1. Spar geometrical parameters and constraints.

\begin{tabular}{|c|c|c|c|c|c|c|}
\hline \multirow{2}{*}{$\begin{array}{l}\text { Spar } \\
\text { Types }\end{array}$} & \multicolumn{6}{|c|}{ Parameters } \\
\hline & $\begin{array}{c}\text { Large } \\
\text { Diameter } \\
(\mathrm{mm})\end{array}$ & $\begin{array}{c}\text { Small } \\
\text { Diameter } \\
(\mathrm{mm})\end{array}$ & $\begin{array}{c}\text { Web } \\
\text { Height } \\
\text { (mm) }\end{array}$ & $\begin{array}{c}\text { Upper } \\
\text { Flange } \\
\text { Thickness }\end{array}$ & $\begin{array}{l}\text { Circular } \\
\text { Hollow } \\
\text { Thickness }\end{array}$ & $\begin{array}{c}\text { Web } \\
\text { Thickness }\end{array}$ \\
\hline I & -- & -- & 120 & -- & -- & -- \\
\hline $\begin{array}{l}\text { Circular } \\
\text { Hollow }\end{array}$ & 140 & 130 & -- & -- & -- & -- \\
\hline & \multicolumn{6}{|c|}{ Constraints } \\
\hline$I$ & 5.7 & -- & $110<\mathrm{h}<120$ & $\begin{array}{c}\mathrm{Ut}<10< \\
20\end{array}$ & -- & -- \\
\hline $\begin{array}{l}\text { Circular } \\
\text { Hollow }\end{array}$ & $140<\mathrm{D}<120$ & $100>d>70$ & -- & -- & $10<\mathrm{Ct}<15$ & $10<\mathrm{Wt}<15$ \\
\hline
\end{tabular}

\subsubsection{Concept of Assembly Generative Modeling approach}

The geometric reference for modeling includes the spar-positioning plane, the sparstarting plane, the spar termination plane, the upper and the lower wing skin surfaces. The geometric reference elements are shown in Figure 5. The spar-positioning plane is used to determine the position of the spar in the wing assembly model. The starting and termination plane of the spar is used to determine the beginning and end of the spar along the spanwise direction.

\subsubsection{Wing Components positioning}

Using SolidWorks design table capabilities, designers can define any number of ribs, spars, and span length. The number, position, and orientation of each element are assigned using lists of parameters published in the input file together with the parameters defining the aircraft outer surfaces. The constraints for wing components assembly confiugrations are relative to wing span and geometry. Table 2 shows constraints of assembly configurations,and Figure 6 shows an example of a generic wing assembly generated by the GM and component positioning.

Table 2. Wing structural assembly constaints.

\begin{tabular}{lll}
\hline Location number & Front Spar & Rear spar \\
\hline Location 1 & $\mathrm{X} * 25 \%$ & $\mathrm{X} * 70 \%$ \\
\hline Location 2 & $X^{*} 30 \%$ & $X^{*} 70 \%$ \\
\hline Location 3 & $\mathrm{X} * 35 \%$ & $\mathrm{X} * 70 \%-75 \%$ \\
\hline Location 4 & $\mathrm{X} * 40 \%$ & $\mathrm{X} 70 \%-80 \%$ \\
\hline
\end{tabular}

Where, $\mathrm{X}$ is chord length and spar locus measures from leading edge. 


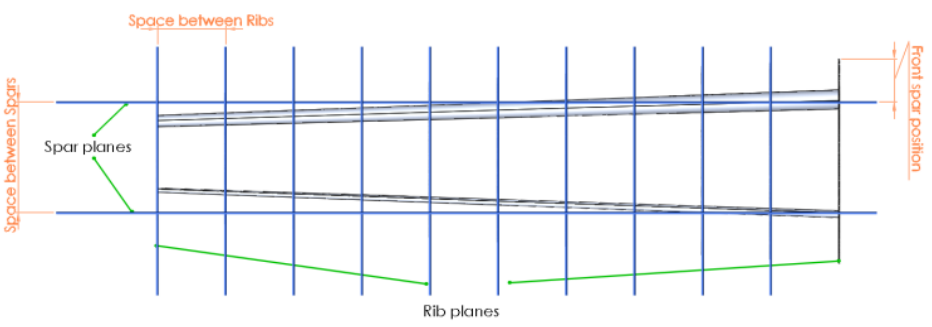

Figure 5. Wing assembly skeleton view and input parameters used to define assembly configurations.

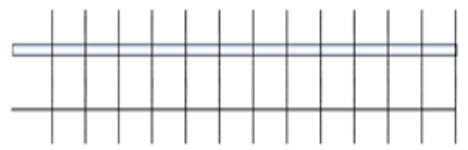

Model 1

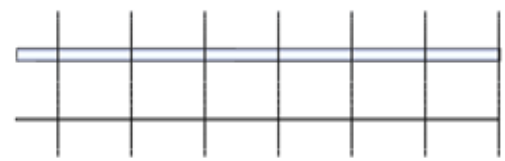

Model 3

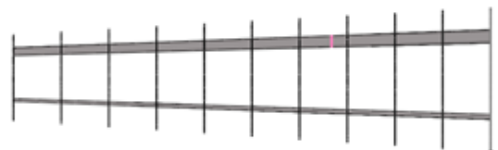

Model 2

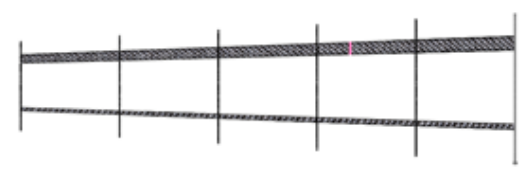

Model 4

Figure 6. Example of generated wing structure configuration.

\subsection{FEA}

The system set up a FE model, starting from a finished CAD model, as produced by the generic design model environment. In this section, a seamless approach is developed that linked the generic model and the FEA environment as shown in Figure 7.

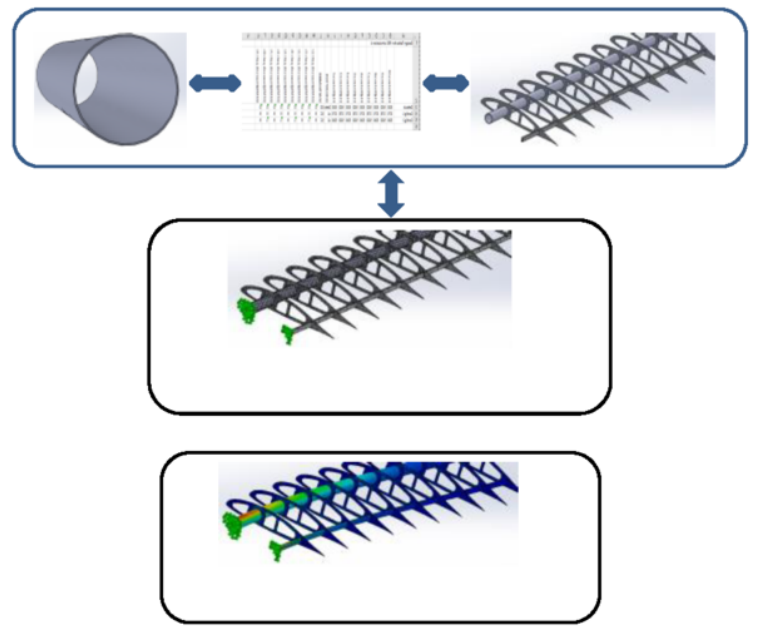

Figure 7. Seamless link between the Generic Model environment and the FEA environment

Geometrical change depends on the configuration in the generic model environment, any selected configuration $\mathrm{CAD}$ will be ready to make mesh generation. Before mesh 
generation dedicated materials are assigned, boundary conditions and forces are applied. Practically, any change that is made in the design table SolidWorks allows the simulation without a need to export the CAD format to another software. In the resulting environment, likely related to two previous environments, mesh update as 3D model changes.

\section{Discussion}

Through the adoption of the automation of generative building systems a designer is able to create a generative model of wing components and wing structural assembly configurations, as per parameters and constraints. Assembly model constraints are set to be relative to wing chord length and span, thus a designer can not exceed a logical positioning and quantity of components under given constraints.

The time to make a change in components and assembly model is one way closer and quickly done by creating several sample models in the design table solution,thus substantially shorter lead-time was achieved to do in repetitive design.

The developed approach system has the capability of automating both CAD models and FE Analysis in the same software without a need of exporting models for further FEM analysis.

When a different design needs to be attempt, the design parameter can be changed and these changes are automatically propagated to the linked models. Hence, a designer is capable to obtain both analytical and simulation results at the same time, likewise, it allows designers to compare models based on design goals and ultimately to make the optimal decision.

\section{Conclusions}

The established system is the first of its kind to use design calculation data acquisition in order to generate both CAD and FEM analysis in similar software.

Some of the significant achievements by the developed system are,

1. Simplistic version of generative modeling for wing components and structure at the preliminary design phase

2. Parametrized CAD model generation

3. Generative finite element mesh generation

4. Faster start-up time for modeling \& FE analysis

5. Easy manipulation of design parameters and constraints

The automating approach entails design calculations and knowledgebase relation to create a CAD model for wing components and assembly. Similarily, capable of automating both CAD models and FE Analysis in the same software without a need of exporting models for further FEM analysis.

When a different design needs to be done, the design parameter can be changed and these changes are automatically propagated to the linked models. Hence, a designer is capable to obtain both analytical and simulation results at the same time, likewise, it allows designers to compare models based on design goals and ultimately to make the optimal decision. This dramatically speeds up the process of geometry generation of different wing planform or configurations so that the designer focuses more on the design of the aircraft wing rather than worrying about the creation of the geometry. 


\section{References}

[1] J.H. McMasters, R.M. Cummings, Rethinking the Airplane Design Process - An Early 21st Century Perspective, AIAA 2004-693, 42nd AIAA Aerospace Sciences Meeting and Exhibit, Reno, NV, USA, Jan. 2004.

[2] J.H. Frazer, Creative design, and the generative evolutionary paradigm, in D.a.B. Corne, Peter, (eds.) Creative evolutionary systems, Morgan Kaufmann Publishers Inc. (Elsevier): California, San Francisco, 2002, pp. 253-274.

[3] N.N., Generative Components, Bentley Systems, 2010; Available from: http://www.bentley.com/enUS/Promo/Generative+Design

[4] N.N., Grasshopper. McNeel Corp. 2010; Available from http://www.grasshopper3d.com/.

[5] N.N., Genometri, Genoform. 2002, Genometri Pte Ltd.: Singapore.

[6] N.N., Para Cloud GEM. 2010; Available from: http://www.paracloud.com/.

[7] K. Shea, R. Aish, and M. Gourtovaia, Towards integrated performance-driven generative Design tools. Automation in Construction, 2005, Vol. 14, Issue 2, March 2005, pp. 253-264.

[8] K. Sivam, A practical generative design method. Computer-Aided Design, 2011, Vol. 43, pp. 88-100.

[9] W.J.C. Verhagen, P. Bermell-Garcia, R.E.C. van Dijk, R. Curran, A Critical Review of Knowledge Based Engineering: An Identification of Research Challenges, Advanced Engineering Informatics, 2012, Vol. 26, Issue 1, pp. 5-15.

[10] E.R. Oxman, Theory, and design in the first digital age. Design Studies, Vol. 27, Issue 3, May 2006, pp. 229-265.

[11] Y. Guoyan, W. Xianozhen and L. Peng, A constrained based evolutionary decision support system for product design, 2009 Chinese Control and Decision Conference, DOI: 10.1109/CCDC.2009.5191831, pp. 2585-2590.

[12] L. Caladas, GENE ARCH: An evolution-based generative design system for sustainable architecture. In: Smith I.F.C. (eds) Intelligent Computing in Engineering and Architecture. EG-ICE 2006. Lecture Notes in Computer Science, vol 4200. Springer, Berlin, Heidelberg. https://doi.org/10.1007/11888598_12.

[13] P.V. Buelov, Using Evolutionarry algorithms to aid designers of architectural structures. In: Creative Evolutionary Systems, P.J.Bentley, Editor. 2002, Morgan Kaufmann San francisco, CA. pp. 315-336

[14] P.J. Bentley, From coffee tables to hospitals: generic evolutionary design, in P.J. Bentley (ed.) Evolutionary Design by Computers, Morgan Kaufmann San Francisco, CA, 1999, pp. 405-423.

[15] J. Huang, A. Pytel, C. Zhang, S. Mann, E. Fourquert, M. Hahn, K. Kinnear, M. Lam and W. Cowan, An Evolution of Shape/Split Grammars for Architecture, Technical Report CS-2009-23. 2009, David R. Cheriton School of Computer Science, University of Waterloo.

[16] K. Amadori, Geometry Based Design Automation - Applied to Aircraft Modelling and Optimization, Linköping Studies in Science and Technology. Dissertations, No. 1418.

[17] S. Thajudeen, M. Lennartsson, F. Elgh, P.J. Persson, Parametric modelling of steel connectors in a glulam based post and beam building system - Towards a flexible product platform approach, Advances in Transdisciplinary Engineering, 2020, Vol. 12, pp. 564-573.

[18] W. Skarka, Application of MOKA methodology in generative model creation using CATIA. Engineering Application of Artificial Intelligence, 2007, Vol. 20, Issue 5, pp. 677-690.

[19] W. Skarka, Using Knowledge-Based Engineering Methods in Designing with Modular Components of Assembly Systems, 11th International Design Conference (Design 2010), May 17-20, 2010, Vol. 1-3, pp. $1837-1846$

[20] A. Jałowiecki, W. Skarka, Generative modeling in ultra-efficient vehicle design, Advances in Transdisciplinary Engineering, 2016, Vol. 4, pp. 999-1008.

[21] C.B. Chapman, M. Pinfold, The application of knowledge-based engineering approach to the rapid design and analysis of an automotive structure, Journal of Advances in Engineering Software, 2001, Vol. 32, Issue 12, pp. 903-912.

[22] W. Skarka, T. Neumann, New methods of designing stamping dies assemblies by using generative models. In: Transdisciplinary engineering: a paradigm shift. Advances in Transdisciplinary Engineering, 2017, Vol. 5, pp. 456-463.

[23] J. Andersson and P. Krus, Metamodel Representations for Robustness Assessment in Multiobjective Optimization, ICED01 International Conference On Engineering Design, Glasgow, UK, Aug. 2001. 